\title{
MAXIMAL LENGTHS OF EXCEPTIONAL COLLECTIONS OF LINE BUNDLES
}

\author{
ALEXANDER I. EFIMOV
}

\begin{abstract}
In this paper we construct infinitely many examples of toric Fano varieties with Picard number three, which do not admit full exceptional collections of line bundles. In particular, this disproves King's conjecture for toric Fano varieties.

More generally, we prove that for any constant $c>\frac{3}{4}$ there exist infinitely many toric Fano varieties $Y$ with Picard number three, such that the maximal length of exceptional collection of line bundles on $Y$ is strictly less than $c$ rk $K_{0}(Y)$. To obtain varieties without exceptional collections of line bundles, it suffices to put $c=1$.

On the other hand, we prove that for any toric nef-Fano DM stack $Y$ with Picard number three, there exists a strong exceptional collection of line bundles on $Y$ of length at least $\frac{3}{4} \mathrm{rk} K_{0}(Y)$. The constant $\frac{3}{4}$ is thus maximal with this property.
\end{abstract}

\section{INTRODUCTION}

A conjecture of King [Ki] claims that each smooth projective toric variety has a full strong exceptional collection of line bundles. It was disproved [HP1, HP2, Mi] in infinitely many cases. However, all the counter-examples were not nef-Fano. Borisov and Hua have proposed the following modification (and a generalization).

Conjecture 1.1. Every smooth nef-Fano toric DM stack possesses a full strong exceptional collection of line bundles.

They proved Conjecture $1.1[\mathrm{BHu}]$ in the case of Fano stacks for which either Picard number or dimension is at most two. The case of nef-Fano Del Pezzo stacks was further treated in [IU].

The weaker version of conjecture was proposed by Costa and Miró-Roig:

Conjecture 1.2. For any smooth, complete Fano toric variety there exists a full, strongly exceptional collection of line bundles.

In this paper we disprove Conjecture 1.2 (and hence Conjecture 1.1) by proving the following theorem.

The author was partially supported by the Moebius Contest Foundation for Young Scientists, and RFBR (grant 4713.2010.1). 
Theorem 1.3. For any constant $c>\frac{3}{4}$ there exist infinitely many toric Fano varieties $Y$ with Picard number three, such that the maximal length of exceptional collection of line bundles on $Y$ is strictly less than $c$ rk $K_{0}(Y)$. In particular (for $c=1$ ), there are infinitely many toric Fano varieties with Picard number three without full exceptional collections of line bundles.

More precise statement is Theorem 6.2. We also give infinitely many explicit examples of toric Fano varieties without full exceptional collections of line bundles (Theorem 6.3). Note that we do not require the collections to be strong.

On the other hand we prove another (positive) result which constructs not full but relatively long, strong exceptional collection of line bundles on each toric nef-Fano DM stack with Picard number three (see Theorem [7.1).

Theorem 1.4. For any toric nef-Fano DM stack $Y$ with Picard number three, there exists a strong exceptional collection of line bundles on $Y$ of length at least $\frac{3}{4} \mathrm{rk} K_{0}(Y)$.

Recall the result of Kawamata:

Theorem 1.5. For any smooth projective toric DM stack $Y$, the derived category $D^{b}(Y)$ is generated by exceptional collection of coherent sheaves.

The following conjecture was suggested to me by D. Orlov.

Conjecture 1.6. For any smooth projective toric DM stack $Y$, the derived category $D^{b}(Y)$ is generated by a strong exceptional collection.

It remains open.

The paper is organized as follows.

In Section 2 we recall some necessary notions and facts about Gale duality.

In Section 3 we recall stacky fans and corresponding toric DM stacks. Here we also describe the conditions on fan corresponding to the (nef-)Fano condition on stack.

Section 4 is devoted to the well-known description of cohomology of line bundles on smooth toric DM stacks (Proposition 4.1). Here we also describe the line bundles with zero cohomology and zero higher cohomology (Corollary 4.2).

Section 5 is devoted to the construction of toric Fano varieties with Picard number three in terms of a Gale dual picture. It is used in the proof of main theorem.

In Section 6 we construct a certain family of toric Fano varieties $Y_{n, k, a}$ parameterized by integers $n, k \geq 2, a \geq 1$. We use varieties of this family to prove Theorem 1.3 (more precisely, Theorem 6.2). The proof is rather technical and contains a lots of technical bounds. Further, using the proof of Theorem 6.2, we prove that varieties $Y_{16, k, 1}$ do not have full exceptional collections of line bundles for $k \geq 386$ (Theorem 6.3). 
In Section 7 we prove Theorem 1.4, The idea is the following. We construct a centrally symmetric polytope $P \subset \operatorname{Pic}_{\mathbb{R}}(Y)$ with the following property: the integral points (treated as line bundles) in the interior of any shift of $\frac{1}{2} P$ form a strong exceptional collection. Further, it follows from Fubini's theorem that for some shift the length of this collection is at least $\frac{1}{8} \operatorname{Vol}(P)$. Then it remains to prove the inequality $\frac{1}{8} \operatorname{Vol}(P) \geq \frac{3}{4} \mathrm{rk} K_{0}(Y)$.

In Appendix we give a combinatorial description of fans defining smooth toric DM stacks with Picard number three.

Acknowledgements. I am grateful to L. Borisov who pointed out a mistake in the proof of Theorem 6.2 in the preliminary version of the paper. Essentially it does not affect the proof, but the smallest dimension of my counter-examples becomes higher. I am also grateful to A. Craw for his remarks and to A. Kuznetsov and D. Orlov for useful discussions.

\section{Gale duality}

In this section we remind some basic notions and facts related to Gale duality.

Let $V$ be a finite-dimensional vector space over $\mathbb{R}$, and $v_{1}, \ldots, v_{n}$ a finite collection of vectors which generate $V$. Then we have the surjection

$$
p: \mathbb{R}^{N} \rightarrow V, e_{i} \mapsto v_{i}, i=1, \ldots, N,
$$

where $e_{1}, \ldots, e_{n}$ are standard basis vectors. Take the dual injection $p^{\vee}: V^{\vee} \hookrightarrow\left(\mathbb{R}^{N}\right)^{\vee} \cong$ $\mathbb{R}^{N}$, and the corresponding quotient map

$$
q: \mathbb{R}^{N} \rightarrow \mathbb{R}^{N} / V^{\vee}=: U
$$

Also put $E_{i}:=q\left(e_{i}\right) \in U, \quad 1 \leq i \leq N$.

Definition 2.1. In the above notation, the surjection (2.2) is called Gale dual to the surjection (2.1). Further, the collection of vectors $E_{1}, \ldots, E_{n} \in U$ is called Gale dual to the collection $v_{1}, \ldots, v_{n} \in V$.

It is clear that the surjection (2.1) is canonically identified with Gale dual to (2.2). Further, it follows from the definition that we have the following isomorphisms:

$$
\begin{aligned}
& \text { \{linear functionals on } V\} \cong\left\{\text { linear relations on } E_{1}, \ldots, E_{n}\right\}, \\
& \{\text { linear functionals on } U\} \cong\left\{\text { linear relations on } v_{1}, \ldots, v_{n}\right\} .
\end{aligned}
$$

For instance, linear functional $l \in V^{\vee}$ gives a linear relation $l\left(v_{1}\right) E_{1}+\cdots+l\left(v_{n}\right) E_{n}=0$.

We would like to reformulate some statements about vectors $v_{i}$ in terms of vectors $E_{i}$. 
Proposition 2.2. The following are equivalent:

(i) the interior of the convex hull of $v_{1}, \ldots, v_{n}$ contains the origin;

(ii) there exists a functional $l \in U^{\vee}$ such that $l\left(E_{i}\right)>0$ for $i=1, \ldots, n$.

Proof. First, (i) is equivalent to the existence of linear relation

$$
a_{1} v_{1}+\cdots+a_{n} v_{n}=0, \quad a_{1}, \ldots, a_{n}>0 .
$$

And this is in turn equivalent to (ii) by (2.4).

Consider the vector $K=-E_{1}-\cdots-E_{n} \in U$. Further, put $\bar{U}:=U /(\mathbb{R} \cdot K)$, and let $\overline{E_{i}} \in \bar{U}$ be the projection of $E_{i} \in U$ for $i=1, \ldots, n$.

Proposition 2.3. Suppose that both equivalent statements in Proposition 2.2 hold. Then the following are equivalent:

(i) points $v_{i} \in V$ are vertices of a convex polytope;

(ii) for each $i=1, \ldots, n$ there exist positive numbers $a_{1}, \ldots, \widehat{a_{i}}, \ldots, a_{n}$ such that $\sum_{j \neq i} a_{j} E_{j}=-K$

(iii) for each $i=1, \ldots, n$, the interior of the convex hull of $\overline{E_{1}}, \ldots, \widehat{\overline{E_{i}}}, \ldots, \overline{E_{n}}$ contains the origin.

Proof. $(i) \Rightarrow(i i)$ Since this convex polytope contains zero (by assumption), for each $i=$ $1, \ldots, N$ there exists a functional $l_{i} \in V^{\vee}$, such that $l_{i}\left(v_{i}\right)=1$ and $l_{i}\left(v_{j}\right)<1$ for $j \neq i$. By (2.3), this functional gives a relation on $E_{j}$ which can be rewritten as follows:

$$
\sum_{j \neq i}\left(1-l_{i}\left(v_{j}\right)\right) E_{j}=-K
$$

The implication is proved.

$(i i) \Rightarrow(i)$ This implication is proved analogously to the previous one.

$(i i) \Rightarrow(i i i)$ We have that $\sum_{j \neq i} a_{j} \overline{E_{j}}=0$ with $a_{j}>0$, hence the assertion.

$($ iii $) \Rightarrow(i i)$ For each $i=1, \ldots, n$ there exists positive numbers $b_{j}, j \neq i$, such that $\sum_{j \neq i} b_{j} \overline{E_{j}}=0$. Then $\sum_{j \neq i} b_{j} E_{j}=a K$ for some $a \in \mathbf{R}$. Take some functional $l \in U^{\vee}$ such that $l\left(E_{j}\right)>0$ for $1 \leq j \leq n$ (such $l$ exists by our assumption). Then

$$
l(a K)=\sum_{j \neq i} b_{j} l\left(E_{j}\right)>0 .
$$

Since $l(K)<0$, we have that $a<0$. Put $a_{j}:=-\frac{b_{j}}{a}, j \neq i$. Then we have $\sum_{j \neq i} a_{j} E_{j}=-K$ and $a_{j}>0$. 
Proposition 2.4. Suppose that all statements of Propositions 2.2 and 2.3 hold. Let $B \subset$ $\{1, \ldots, n\}$ be a non-empty subset, and $\bar{B} \subset\{1, \ldots, n\}$ its complement. Then the following are equivalent:

(i) the set $\left\{v_{j}, j \in B\right\}$ is the set of vertices of some face of the polytope;

(ii) there exist positive numbers $a_{j}, j \in \bar{B}$ such that $\sum_{j \in \bar{B}} a_{j} E_{j}=-K$;

(iii) the relative interior of the convex hull of $\bar{E}_{j}, j \in \bar{B}$, contains the origin.

Proof. The proof goes absolutely analogously to Proposition 2.3 ,

Recall that a convex polytope is called simplicial if all its facets (and hence all faces) are simplices. We have the following corollary.

Corollary 2.5. Suppose that all statements of Propositions 2.2 and 2.3 hold. Then the following are equivalent:

(i) the convex hull of $v_{1}, \ldots, v_{n}$ is a simplicial polytope;

(ii) for any subset $A \subset\{1, \ldots, N\}$ with $|A|<\operatorname{dim} U=N-\operatorname{dim} V$, the convex hull of $\overline{E_{j}}, j \in A$, does not contain the origin.

Now note that we have natural isomorphism $\operatorname{det}(V) \cong \operatorname{det}\left(\mathbb{R}^{n} / V^{\vee}\right)=\operatorname{det}(U)$ of onedimensional spaces. Fix some volume forms $\omega$ on $V$ and $\omega^{\prime}$ on $U$ which correspond to each other.

Lemma 2.6. Choose a permutation $\sigma \in S_{n}$. Then we have

$$
\left|\omega\left(v_{\sigma(1)}, \ldots, v_{\sigma(\operatorname{dim} V)}\right)\right|=\left|\omega^{\prime}\left(E_{\sigma(\operatorname{dim} V+1)}, \ldots, E_{\sigma(n)}\right)\right| .
$$

Proof. We may and will assume that $\sigma=$ id. Take the dual vollume form $\omega^{\vee}$ on $V^{\vee}$ and choose functionals $f_{1}, \ldots, f_{\operatorname{dim} V} \in V^{\vee}$ such that $\omega^{\vee}\left(f_{1}, \ldots, f_{\operatorname{dim} V}\right)=1$. Then we have the following chain of equalities:

$$
\begin{aligned}
\left|\omega\left(v_{1}, \ldots, v_{\operatorname{dim} V}\right)\right| & =\left|\omega\left(v_{1}, \ldots, v_{\operatorname{dim} V}\right)\right| \cdot\left|\omega^{\vee}\left(f_{1}, \ldots, f_{\operatorname{dim} V}\right)\right|=\left|\operatorname{det}\left(f_{i}\left(v_{j}\right)\right)_{1 \leq i, j \leq \operatorname{dim} V}\right| \\
& =\left|\operatorname{det}\left(p^{\vee}\left(f_{1}\right), \ldots, p^{\vee}\left(f_{\operatorname{dim} V}\right), e_{\operatorname{dim} V+1}, \ldots, e_{n}\right)\right| \\
= & \left|\omega^{\vee}\left(f_{1}, \ldots, f_{\operatorname{dim} V}\right)\right| \cdot\left|\omega^{\prime}\left(E_{\operatorname{dim} V+1}, \ldots, E_{n}\right)\right|=\left|\omega^{\prime}\left(E_{\operatorname{dim} V+1}, \ldots, E_{n}\right)\right| .
\end{aligned}
$$

Lemma is proved.

Note that Gale duality can be also considered for integer lattices so that after tensoring with $\mathbb{R}$ we obtain the above picture. More precisely, we do not assume that $v_{1}, \ldots, v_{n} \in C$ generate the lattice $C$ but we still assume that they generate the real space $C_{\mathbb{R}}$. The Gale dual collection $E_{1}, \ldots, E_{n} \in D$ generates the abelian group $D$. It may have torsion:

$$
D_{\text {tors }} \cong \operatorname{Hom}\left(C /\left(\mathbb{Z} \cdot v_{1}+\cdots+\mathbb{Z} \cdot v_{n}\right), \mathbb{C}^{*}\right) .
$$


Lemma 2.7. Assume that $V=C_{\mathbb{R}}$, where $C$ is some integral lattice, and we have $v_{i} \in C$. Choose vollume form $\omega$ in such a way that the volume of unit parallelepiped of $C$ equals to 1. Let $D$ be a Gale dual lattice. Then the volume of unit parallelepiped of $D / D_{\text {tors }}$ (with respect to $\omega^{\prime}$ on $D_{\mathbb{R}}=U$ ) equals to

$$
\left|D_{\text {tors }}\right|
$$

Proof. We may and will assume that $D_{\text {tors }}=0$ (by replacing $C$ with $\mathbb{Z} \cdot v_{1}+\cdots+\mathbb{Z} \cdot v_{n}$ and $\omega$ with $\left|D_{\text {tors }}\right| \cdot \omega$, according to (2.5) ). Choose any basis $f_{1}, \ldots, f_{\operatorname{dim} V}$ of $\operatorname{Hom}(C, \mathbb{Z})$, and choose any $u_{1}, \ldots, u_{\operatorname{dim} U} \in \mathbb{Z}^{N}$ such that $q\left(u_{1}\right), \ldots, q\left(u_{\operatorname{dim} U}\right)$ form a basis of $D$. Then

$$
\left|\omega^{\prime}\left(q\left(u_{1}\right), \ldots, q\left(u_{\operatorname{dim} U}\right)\right)\right|=\left|\operatorname{det}\left(u_{1}, \ldots, u_{\operatorname{dim} U}, p^{\vee}\left(f_{1}\right), \ldots, p^{\vee}\left(f_{\operatorname{dim} V}\right)\right)\right|=1
$$

since $p^{\vee}\left(f_{1}\right), \ldots, p^{\vee}\left(f_{\operatorname{dim} V}\right), u_{1}, \ldots, u_{\operatorname{dim} U}$ generate $\mathbb{Z}^{N}$.

We have the following

Corollary 2.8. Let $v_{1}, \ldots, v_{n} \in C$ be a collection of vectors in integer lattice which generate it. Let $E_{1}, \ldots, E_{n} \in D$ be a Gale dual collection. Further, let $A \subset\{1, \ldots, N\}$ be a subset with $|A|=\operatorname{rank}(C)$, and $\bar{A} \subset\{1, \ldots, N\}$ its complement. Then the following are equivalent:

(i) the vectors $v_{j}, j \in A$, generate the lattice $C$;

(ii) the vectors $E_{j}, j \in \bar{A}$, generate the lattice $D$.

Proof. This follows immediately from Lemmas 2.6 and 2.7.

\section{Smooth Toric DM STACKS}

Let $N$ be a free finitely generated abelian group, and let $\Sigma$ be a complete simplicial fan in $N$. We call $\Sigma$ a stacky fan if on any one-dimensional cone $\sigma \in \Sigma(1)$ there chosen a non-zero vector $v_{\sigma} \in \sigma \cap N$.

The associated toric DM stack $Y=Y_{\Sigma}$ is constructed as follows. We have natural surjection

$$
\mathbb{Z}^{\Sigma(1)} \rightarrow N, \quad e_{\sigma} \mapsto v_{\sigma}
$$

Put

$$
\operatorname{Gale}(N):=\operatorname{Coker}\left(N^{\vee} \rightarrow \mathbb{Z}^{\Sigma(1)}\right) .
$$

Define the algebraic Group $G$ by the formula

$$
G:=\operatorname{Hom}\left(\operatorname{Gale}(N), \mathbb{C}^{*}\right) .
$$

Define the open subset $U \subset \mathbb{C}^{\Sigma(1)}$ as follows. The point $z \in \mathbb{C}^{\Sigma(1)}$ lies in $U$ if the set $\left\{\sigma \in \Sigma(1) \mid z_{\sigma}=0\right\}$ is not a set of one-dimensional cones of some cone in $\Sigma$. 
We have a natural action of $G$ on $U$ via inclusion $G \subset\left(\mathbb{C}^{*}\right)^{\Sigma(1)}$. Put

$$
Y_{\Sigma}:=U / G
$$

The stack $Y_{\Sigma}$ is smooth and complete. The torus

$$
T=\left(\mathbb{C}^{*}\right)^{\Sigma(1)} / G
$$

naturally acts on $Y_{\Sigma}$. The orbits of codimension $i$ are in bijection with cones $\sigma \in \Sigma(i)$ :

$$
\sigma \leftrightarrow\left\{z \in U \mid z_{l}=0 \text { for } l \subset \partial \sigma, \quad z_{l} \neq 0 \text { for } l \not \subset \partial \sigma\right\} / G .
$$

If in addition for each maximal cone the vectors $v_{\sigma}$ on its boundary form a basis of $N$, then $Y_{\Sigma}$ is just a toric variety. The following is well-known (see [FLTZ], Theorem 4.4):

Proposition 3.1. The stack $Y_{\Sigma}$ is nef-Fano (resp. Fano) iff the polytope

$$
\bigcup_{\left\langle v_{i_{1}}, \ldots, v_{i_{\mathrm{rk} N}}\right\rangle \in \Sigma(\mathrm{rk} N)} \operatorname{conv}\left(v_{i_{1}}, \ldots, v_{i_{\operatorname{dim} Y}}, 0\right)
$$

is convex (resp. in addition all $v_{\sigma}$ are its vertices and it is simplicial).

We will also need the following formula for the rank of $K_{0}\left(Y_{\Sigma}\right)[\mathrm{BHo}$ :

$$
\operatorname{rk} K_{0}\left(Y_{\Sigma}\right)=(\operatorname{rk} N) ! \operatorname{Vol}\left(\underset{\left\langle i_{i_{1}}, \ldots, v_{i_{\mathrm{rk} N}}\right\rangle \in \Sigma(\operatorname{rk} N)}{\bigcup} \operatorname{conv}\left(v_{i_{1}}, \ldots, v_{i_{\operatorname{dim} Y}}, 0\right)\right) .
$$

In particular, if $Y_{\Sigma}$ is a variety, then $\operatorname{rk} K_{0}\left(Y_{\Sigma}\right)$ equals to the number of maximal cones in $\Sigma$ (or, equivalently, torus-invariant points in $Y_{\Sigma}$ ).

\section{Cohomology of line Bundles on Smooth toric DM stacks}

Let $\Sigma$ be a complete simplicial stacky fan, and $Y=Y_{\Sigma}$ the corresponding stack. We have that

$$
\operatorname{Pic}(Y)=\operatorname{Pic}_{G}(U)=\operatorname{Hom}\left(G, \mathbb{C}^{*}\right) \cong \operatorname{Gale}(N) .
$$

Denote by $\left\{\mathcal{O}\left(E_{\sigma}\right) \in \operatorname{Pic}(Y)\right\}_{\sigma \in \Sigma(1)}$ the Gale dual collection to $\left\{v_{\sigma} \in N\right\}_{\sigma \in \Sigma(1)}$. Then $\mathcal{O}\left(E_{\sigma}\right)$ is a line bundle of invariant divisor corresponding to $\sigma$. In the next sections we will not distinguish divisors and the corresponding line bundles.

Further, for any $I \subset \Sigma(1)$ denote by $C_{I}$ the simplicial complex with the vertex set $I$, which consists of subsets $J \subset I$ which are precisely boundary cones of some cone in $\Sigma$. For instance, $\left|C_{\emptyset}\right|=\emptyset$ and $\left|C_{\Sigma(1)}\right|$ is homeomorphic to $S^{\mathrm{rk} N-1}$.

Also, for any $r \in \mathbb{Z}^{\Sigma(1)}$, put

$$
\operatorname{Supp}(r)=\left\{\sigma \in \Sigma(1) \mid r_{\sigma}<0\right\} .
$$


The following is well-known (computation by Čech).

Proposition 4.1. Let $L$ be a line bundle on $Y$. Then

$$
H^{i}(L)=\bigoplus_{\mathcal{O}\left(\sum_{\sigma \in \Sigma(1)}^{r \in \mathbb{Z}^{\Sigma(1)}} r_{\sigma} E_{\sigma}\right) \cong L} \bar{H}_{i-1}\left(\left|C_{\operatorname{Supp}(r)}\right|\right) .
$$

For any $I \subset \Sigma(1)$ such that $\bar{H} \cdot\left(\left|C_{I}\right|\right) \neq 0$, we put

$$
K_{I}:=\left\{\mathcal{O}\left(\sum_{\sigma \in I}\left(-r_{\sigma}-1\right) E_{\sigma}+\sum_{\sigma \notin I} r_{\sigma} E_{\sigma}\right) \mid r_{\sigma} \in \mathbb{Z}_{\geq 0}, \sigma \in \Sigma(1)\right\} \subset \operatorname{Pic} Y .
$$

We call such $K_{I}$ forbidden sets. For instance, $K_{\emptyset}$ is the set of all effective line bundles.

Corollary 4.2. Let $L$ be a line bundle on $Y$. The following are equivalent:

(i) $H^{\cdot}(L)=0 \quad$ (resp. $\left.H^{>0}(L)=0\right)$;

(ii) $L$ does not belong to any forbidden $K_{I}$ (resp. to any forbidden $K_{I}, \quad I \neq \emptyset$ ).

Proof. This follows immediately from Proposition 4.1 .

We will need the notion of a primitive collection.

Definition 4.3. A non-empty subset $I \subset \Sigma(1)$ is called a primitive collection if it is not a set of boundary cones of any cone in $\Sigma$, but each proper subset $J \subset I$ is.

Note that primitive collections describe the combinatorial structure of a fan (i.e. the corresponding simplicial complex).

Lemma 4.4. Let $I \subset \Sigma(1)$ be a non-empty subset such that $\bar{H}^{\text {cdot }}\left(\left|C_{I}\right|\right) \neq 0$. Then $I$ is a union of primitive collections.

Proof. Consider the equivariant Picard group $\operatorname{Pic}_{T}(Y) \cong \mathbb{Z}^{\Sigma(1)}$, with basis given by $\mathcal{O}\left(E_{i}\right)$ with obvious equivariant structures. Then a computation by Čech shows that

$$
H_{T}^{i}\left(\mathcal{O}\left(\sum_{\sigma \in \Sigma(1)} r_{\sigma} E_{\sigma}\right)\right) \cong \bar{H}_{i-1}\left(\left|c_{\operatorname{Supp}(r)}\right|\right) .
$$

Now consider our subset $I$. Take some element $i \in I$. We need to prove that there exists a primitive collection $J \subset I$ such that $i \in J$. If $I$ is a primitive collection itself, then there is nothing to prove. Otherwise, there exists some proper subset $J \subset I$ which is a primitive collection.

If $i \in J$, then we are done. Otherwise, the (twisted by $\mathcal{O}\left(-E_{i}\right)$ ) Koszul complex

$$
\mathcal{O}\left(-E_{i}\right) \otimes \bigotimes_{j \in I \backslash\{i\}}\left(\mathcal{O}\left(-E_{j}\right) \rightarrow \mathcal{O}\right)
$$


of $T$-equivariant vector bundles is acyclic. Since

$$
H^{>0}\left(\mathcal{O}\left(-\sum_{j \in I} E_{j}\right)\right) \cong \bar{H} \cdot\left(\left|C_{I}\right|\right) \neq 0
$$

we have that for some proper $J \subset I$ containing $i$,

$$
H^{>0}\left(\mathcal{O}\left(-\sum_{j \in I} E_{j}\right)\right) \cong \bar{H} \cdot\left(\left|C_{I}\right|\right) \neq 0 .
$$

We may replace $I$ by $I^{\prime}$. Iterating, we will come to some primitive $I^{\prime \prime} \subset I$ containing $i$. This proves Lemma.

\section{Toric Fano VARieties With Picard number three}

Let $\Sigma$ be a stacky fan in some integer lattice $C$, such that the corresponding stack $Y:=Y_{\Sigma}$ is a Fano variety with Picard number three. By the description of Batyrev [Ba], there exists a decomposition $\Sigma(1)=X_{0} \sqcup \cdots \sqcup X_{2 t}$ with $t \in\{1,2\}$ and $X_{i} \neq \emptyset$, such that primitive collections in $\Sigma(1)$ are precisely $X_{i} \cup \cdots \cup X_{i+t-1}, \quad 0 \leq i \leq 2 t$, where we put $X_{i+2 t+1}:=X_{i}$. In the case $t=1$, the King' conjecture was proved (more generally, it was proved for all projective toric varieties with disjoint primitive collections) [CM], Theorem 1.3 .

We will deal with the case $t=2$. Note that complements to maximal cones are precisely sets of the form $\{p, q, r\}$, where for some $i p \in X_{i}, q \in X_{i+1}, r \in X_{i+3}$. Hence, by (3.1), we have the following formula for the rank of $K_{0}(Y)$.

$$
\operatorname{rk} K_{0}(Y)=\sum_{i=0}^{4}\left|X_{i}\right| \cdot\left|X_{i+1}\right| \cdot\left|X_{i+3}\right| .
$$

Let $E_{i} \in \operatorname{Pic} Y, \quad i \in \Sigma(1)$, be invariant divisors corresponding to one-dimensional cones. Note that they determine the vectors $v_{i}$ via Gale duality. Further, the vectors $v_{i}$ determine the fan by the Fano condition (Proposition 3.1).

Proposition 5.1. Let $E_{1}, \ldots, E_{N} \in \mathbb{Z}^{3}$ be a collection of vectors which generate the lattice. Suppose that the following conditions hold:

1) There exists a functional $l \in\left(\mathbb{R}^{3}\right)^{\vee}$ such that $l\left(E_{j}\right)>0$ for $j=1, \ldots, N$;

2) There exists a decomposition $\{1, \ldots, N\}=X_{0} \sqcup \cdots \sqcup X_{4}$ (the numeration is cyclic), $X_{i} \neq \emptyset$, and functionals $l_{0}, \ldots, l_{4} \in\left(\mathbb{R}^{3}\right)^{\vee}$ such that

$$
l_{i}\left(E_{j}\right) \begin{cases}>0 & \text { for } j \in X_{i} \cup X_{i+1} \\ <0 & \text { otherwise }\end{cases}
$$

and $l_{i}\left(E_{1}+\cdots+E_{N}\right)=0$. 
3) For each $i=0, \ldots, 4$, all triples $p \in X_{i}, q \in X_{i+1}, r \in X_{i+3}$, the vectors $E_{p}, E_{q}, E_{r}$ form a basis of $\mathbb{Z}^{3}$.

Then the Gale dual collection of vectors defines a toric Fano variety with five primitive collections $X_{i} \cup X_{i+1}$.

Proof. Let $v_{1}, \ldots, v_{n} \in \mathbb{Z}^{N-3}$ be the Gale dual collection.

By condition 1) and Proposition [2.2, the interior of the convex hull of $v_{i}$ contains the origin.

Put $K:=-E_{1}-\cdots-E_{N}, W:=\mathbb{R}^{3} /(\mathbb{R} \cdot K)$, and let $\overline{E_{i}} \in W$ be the projections of $E_{i}$. It follows from condition 2) that the following are equivalent:

(i) The interior of the convex hull of $\overline{E_{p}}, \overline{E_{q}}, \overline{E_{r}}$ contains zero;

(ii) for some $0 \leq i \leq 4$ and permutation of $p, q, r$, we have $p \in X_{i}, q \in X_{i+1}, r \in X_{i+3}$.

Hence, by Proposition [2.3, the points $v_{i}$ are vertices of a convex polytope. Again by condition 2), for any $1 \leq k<l \leq N$ the convex hull of $\overline{E_{k}}, \overline{E_{l}}$ does not contain the origin. Hence, our convex polytope is simplicial. Further, by equivalence $(i) \Leftrightarrow(i i)$ and Proposition 2.4, the complements to (sets of vertices of) facets are of the form $\{p, q, r\}$, $p \in X_{i}, q \in X_{i+1}, \quad r \in X_{i+3}$. Hence, by condition 3) and Corollary 2.8, the vertices of each facet generate the lattice. Therefore, the vectors $v_{i}$ define a fan describing toric Fano variety. From the description of maximal cones, we see that primitive collections are precisely $X_{i} \cup X_{i+1}, \quad i \in \mathbb{Z} / 5$.

\section{Construction of varieties}

In this section we define a family of toric Fano varieties with Picard number three, parameterized by three positive integers. We will use it to prove the main theorem.

Take some integers $n \geq 2, k \geq 2, a \geq 1$. We define five collections of vectors in $\mathbb{Z}^{3}$ :

1) $\left|X_{0}\right|=n+2 a, \quad E_{0,1}=\cdots=E_{0, n+2 a}=(1,0,0)$;

2) $\left|X_{1}\right|=1, \quad E_{1,1}=(0,1,0)$;

3) $\left|X_{2}\right|=k, \quad E_{2,1}=\cdots=E_{2, k-1}=(0,1,1), \quad E_{2, k}=(-a, 1,1)$;

4) $\left|X_{3}\right|=n, \quad E_{3,1}=\cdots=E_{3, n-1}=(0,0,1), \quad E_{3, n}=(-a, 0,1)$;

5) $\left|X_{4}\right|=1, \quad E_{4,1}=(1,-1,0)$.

Proposition 6.1. For any $n, k, a$, the Gale dual collection to $X_{0} \cup \cdots \cup X_{4}$ defines a toric Fano variety with five primitive collections $X_{i} \cup X_{i+1}$.

Proof. We will just apply Proposition 5.1 and check that all required conditions are satisfied.

First, take the functional $x+\frac{y}{2}+(a+1) z$. It is positive on each $E_{i, j}$, hence the condition 1) is satisfied. 
Further, we have $-K:=\sum_{i, j} E_{i, j}=(n+1, k, k+n)$. Take the projection

$$
\pi: \mathbb{R}^{3} \rightarrow \mathbb{R}^{2} \cong \mathbb{R}^{3} /(\mathbb{R} \cdot K), \quad \pi(x, y, z)=((k+n) x-(n+1) z,(k+n) y-k z),
$$

and put $\overline{E_{i, j}}:=\pi\left(E_{i, j}\right)$. Then we have:

$$
\begin{aligned}
& \overline{E_{0,1}}=\cdots=\overline{E_{0, n+2 a}}=(k+n, 0) ; \\
& \overline{E_{1,1}}=(0, k+n) . \\
& \overline{E_{2,1}}=\cdots=\overline{E_{2, k-1}}=(-n-1, n), \overline{E_{2, k}}=(-k a-n a-n-1, n) ; \\
& \overline{E_{3,1}}=\cdots=\overline{E_{3, n-1}}=(-n-1,-k), \overline{E_{3, n}}=(-k a-n a-n-1,-k) \\
& \overline{E_{4,1}}=(k+n,-k-n) .
\end{aligned}
$$

The required functionals $l_{i} \in\left(\mathbb{R}^{3}\right)^{\vee}$ can be defined as pullbacks: $l_{i}=\pi^{*}\left(f_{i}\right)$, where

$f_{0}=2 n x+(2 n+1) y$

$f_{1}=-x+(k a+n a+n+2) y$;

$f_{2}:=-2 x-y$;

$f_{3}:=-x-(k a+n a+n+2) y$

$f_{4}:=k x-y$.

Thus, the condition 2) is also satisfied. Finally, condition 3) is checked straightforwardly by computing the determinants.

Denote by $Y_{n, k, a}$ the toric Fano variety which is obtained from the above Proposition. For completeness, we describe explicitly the corresponding fan $\Sigma_{n, k, a}$. To obtain the Gale dual collection to $E_{i, j}$, we choose a basis of additive relations on $E_{i, j}$ :

$$
\begin{gathered}
E_{0,1}-E_{1,1}-E_{4,1}=0 \\
E_{0, j}-E_{0, j+1}=0, \quad 1 \leq j \leq n+2 a-1 ; \\
E_{1,1}-E_{2, k}+E_{3, n}=0 \\
E_{2, j}-E_{2, j+1}=0, \quad 1 \leq j \leq k-2 ; \\
E_{2, k-1}-E_{2, k}-E_{3, n-1}+E_{3, n}=0 ; \\
E_{3, j}-E_{3, j+1}=0, \quad 1 \leq j \leq n-2 ; \\
a E_{2, k-1}-(a+1) E_{3, n-1}+E_{3, n}+a E_{4,1}=0 .
\end{gathered}
$$

Now, the Gale dual collection $v_{i, j}$ in $\mathbb{Z}^{2 n+2 a+k-1}$ is the following:

$$
\begin{gathered}
v_{0,1}=e_{1}+e_{2}, \quad v_{0, i}=e_{i+1}-e_{i}, \quad 2 \leq i \leq n+2 a-1, \quad v_{0, n+2 a}=-e_{n+2 a} ; \\
v_{1,1}=e_{n+2 a+1}-e_{1} ; \\
v_{2,1}=e_{n+2 a+2} \text { if } k \geq 3, \quad v_{2, i}=e_{n+2 a+i+1}-e_{n+2 a+i}, \quad 2 \leq i \leq k-2, \\
v_{2, k-1}=e_{n+2 a+k}+a e_{2 n+2 a+k-1}-e_{n+2 a+k-1}, \quad v_{2, k}=-e_{n+2 a+1}-e_{n+2 a+k} ; \\
v_{3,1}=e_{n+2 a+k+1} \text { if } n \geq 3, \quad v_{3, i}=e_{n+2 a+k+i}-e_{n+2 a+k+i-1}, \quad 2 \leq i \leq n-2,
\end{gathered}
$$




$$
\begin{aligned}
& v_{3, n-1}= \begin{cases}-e_{n+2 a+k}-e_{2 n+2 a+k-2}-(a+1) e_{2 n+2 a+k-1} & \text { if } n \geq 3 ; \\
-e_{n+2 a+k}-(a+1) e_{2 n+2 a+k-1} & \text { if } n=2 ;\end{cases} \\
& v_{3, n}=e_{n+2 a+1}+e_{n+2 a+k}+e_{2 n+2 a+k-1}, \quad v_{4,1}=a e_{2 n+2 a+k-1}-e_{1} .
\end{aligned}
$$

The vectors $v_{i, j}$ are vertices of some convex simplicial polytope $Q \subset \mathbb{R}^{2 n+2 a+k-1}$ containing zero. The maximal cones of $\Sigma_{n, k, a}$ are cones over the facets of $Q$. The sets of vertices of the facets are precisely the complements to the sets of the form

$$
\left\{v_{i, j_{1}}, v_{i+1, j_{2}}, v_{i+3, j_{3}}\right\} .
$$

This describes the fan $\Sigma_{n, k, a}$ completely.

For any variety $Y$ with exceptional structure sheaf denote by $l(Y)$ the maximal length of exceptional collections of line bundles. Clearly, if a variety $Y$ has a full exceptional collection of line bundles, then $K_{0}(Y) \cong \mathbb{Z}^{l(Y)}$.

We will prove the following result:

Theorem 6.2. For any constant $c>\frac{3}{4}$ and any $a \in \mathbb{Z}_{>0}$ there exist $n_{0}(a, c) \in \mathbb{Z}_{>0}$ such that for any $n \geq n_{0}(a, c)$ there exists $k_{0}(n, a, c) \in \mathbb{Z}_{>0}$ such that for any $k \geq k_{0}(n, a, c)$ we have

$$
l\left(Y_{n, k, a}\right)<c \operatorname{rk} K_{0}\left(Y_{n, k, a}\right) .
$$

Clearly, to obtain toric Fano's without exceptional collections of line bundles, it suffices to take $c=1$. In this case we have the following explicit result:

Theorem 6.3. Let $a=1, n=16, k \geq 386$. Then

$$
l\left(Y_{n, k, a}\right)<\operatorname{rk} K_{0}\left(Y_{n, k, a}\right) .
$$

In particular, there does not exist a full exceptional collection of line bundles on $Y_{n, k, a}$

We denote by $K=K_{Y_{n, k, a}}=-\sum_{i, j} E_{i, j}$ the canonical class of $Y_{n, k, a}$. For each $i \in \mathbb{Z} / 5 \mathbb{Z}$ we denote by $K_{i} \subset \operatorname{Pic} Y_{n, k, a}$ the forbidden set corresponding to $X_{i} \cup X_{i+1} \cup X_{i+2}$, and by $\widehat{K_{i}}$ the forbidden set corresponding to $X_{i+3} \cup X_{i+4}$, so that $K_{i}=K-\widehat{K}_{i}$. Further, we denote by $K_{\text {eff }} \subset \operatorname{Pic} Y_{n, k, a}$ the set of effective line bundles, and by $K_{n e g}$ the forbidden set corresponding to $\Sigma(1)$, so that $K_{n e g}=K-K_{\text {eff }}$. Also, we put

$$
K_{\text {all }}=\bigcup_{i \in \mathbb{Z} / 5 \mathbb{Z}}\left(K_{i} \cup \widehat{K_{i}}\right) \cup K_{\text {eff }} \cup K_{\text {neg }} .
$$

Clearly, $K_{\text {all }}=K-K_{\text {all }}$. We use identification $\operatorname{Pic} Y_{n, k, a} \cong \mathbb{Z}^{3}$ (by our definition of $\left.Y_{n, k, a}\right)$. 
Proof of Theorem 6.2. Suppose that $\left(L_{1}, L_{2}, \ldots, L_{m}\right)$ is an exceptional collection of line bundles on $Y_{n, k, a}$. Denote the coordinates of $L_{i}$ by $\left(x_{i}, y_{i}, z_{i}\right)$. Then we have

$$
\left(x_{i}-x_{j}, y_{i}-y_{j}, z_{i}-z_{j}\right) \notin K_{\text {all }}, \quad \text { for } 1 \leq i<j \leq m .
$$

Our proof consists of several steps.

Step 1. First we prove the following bound for the maximal difference between $z_{i}$ :

$$
\max \left(z_{i}\right)-\min \left(z_{i}\right) \leq n+k+\left\lceil\frac{n}{a}\right\rceil+1 .
$$

Straightforward computation shows that the following holds:

$$
\begin{gathered}
K_{e f f}=\{(x, y, z) \mid z \geq 0, x \geq-a z+\max (-y, 0)\} ; \\
\widehat{K_{1}}=\{(x, y, z) \mid y \geq 1, z \geq 0, x \leq-n-2 a-1\} ; \\
\widehat{K_{2}}=\{(x, y, z) \mid y \leq z-1, z \geq 0, x \leq-n-2 a-1+z-y\} .
\end{gathered}
$$

From (6.3), (6.4) and (6.5) we conclude that

$$
\left\{z \geq\left\lceil\frac{n}{a}\right\rceil+2\right\} \subset K_{\text {eff }} \cup \widehat{K_{1}} \cup \widehat{K_{2}} \subset K_{\text {all }} .
$$

By the central symmetry, we have that

$$
\left\{z \leq-n-k-\left\lceil\frac{n}{a}\right\rceil-2\right\} \subset K_{n e g} \cup K_{1} \cup K_{2} \subset K_{\text {all }} .
$$

Combining (6.1) with (6.6) and (6.7), we obtain the desired estimate (6.2). We may and will assume that $\max \left(z_{i}\right)=0$. Then each of $z_{i}$ belongs to the interval $\left[-n-k-\left\lceil\frac{n}{a}\right\rceil-1,0\right]$. Step 2. Now choose some $\epsilon>0$ and consider the following functions:

$$
y_{\max }(z)=\max \left(\left\{y_{i} \mid z_{i}=z\right\}\right), \quad y_{\min }(z)=\min \left(\left\{y_{i} \mid z_{i}=z\right\}\right),
$$

which are defined for those $z$ for which there exist $i$ with $z_{i}=z$. We put

$$
T_{\epsilon}=\#\left\{z \mid y_{\max }(z)-y_{\min }(z)>n(1+\epsilon)\right\} .
$$

Our goal is to prove the following upper bound on $T_{\epsilon}$ :

$$
T_{\epsilon}<\frac{\left(\left\lceil\frac{n}{a}\right\rceil+2\right)(k+\epsilon n)}{\epsilon n\left(n+k+\left\lceil\frac{n}{a}\right\rceil+2\right)} .
$$

According to (16.2), the functions $y_{\max }$ and $y_{\min }$ are defined for at most $\left(n+k+\left\lceil\frac{n}{a}\right\rceil+2\right)$ values of $z$. Hence, by the Dirichlet's principle, there exists a residue $d \in \mathbb{Z} /\left(\left\lceil\frac{n}{a}\right\rceil+2\right) \mathbb{Z}$ such that

$$
\#\left\{z \equiv d \bmod \left(\left\lceil\frac{n}{a}\right\rceil+2\right) \mid y_{\max }(z)-y_{\min }(z)>n(1+\epsilon)\right\} \geq \frac{T_{\epsilon}\left(n+k+\left\lceil\frac{n}{a}\right\rceil+2\right)}{\left\lceil\frac{n}{a}\right\rceil+2} .
$$

Fix such $d$, and denote by $T_{d}$ the LHS of (6.10). 
We need another four forbidden sets written explicitly:

$$
\begin{gathered}
K_{0}=\{(x, y, z) \mid y \leq \min (-k-1, z-1), x \leq-a(y+k)-n-2 a\} ; \\
\widehat{K_{0}}=\{(x, y, z) \mid y \geq \max (1, z+n+1), x \geq-a y+2 a-1\} ; \\
K_{3}=\{(x, y, z) \mid y \geq \max (z+n+1,1), x \leq a(y-z-n-1)-n-a-1\} ; \\
\widehat{K_{3}}=\{(x, y, z) \mid y \leq \min (z-1,-k-1), x \geq a(y-z)+2 a\} .
\end{gathered}
$$

Then it is easy to see that

$$
\begin{gathered}
\{(x, y, z) \mid z \leq 0, y \geq n+1\} \subset \widehat{K_{0}} \cup K_{3}, \\
\{(x, y, z) \mid z \geq-n-k, y \leq-n-k-1\} \subset K_{0} \cup \widehat{K_{3}} .
\end{gathered}
$$

Now let

$$
\left\{r_{1}, \ldots, r_{T_{d}}\right\}=\left\{z \equiv d \bmod \left(\left\lceil\frac{n}{a}\right\rceil+2\right) \mid y_{\max }(z)-y_{\min }(z)>n(1+\epsilon)\right\},
$$

where $r_{1}<r_{2}<\cdots<r_{T_{d}}$. From the definition of $r_{i}$ it follows that $r_{i}-r_{j} \geq\left\lceil\frac{n}{a}\right\rceil+2$ for $i>j$. Hence, from (6.6) we conclude that equalities $z_{s}=r_{i}, z_{w}=r_{j}$ imply that $\operatorname{Sign}(s-w)=\operatorname{Sign}(i-j)$. Thus, inclusion (6.15) implies that

$$
y_{\max }\left(r_{i}\right)-y_{\min }\left(r_{i+1}\right) \leq n, \quad 1 \leq i \leq T_{d}-1 .
$$

By definition of $r_{i}$, we obtain

$$
y_{\max }\left(r_{i}\right)-y_{\max }\left(r_{i+1}\right)<y_{\max }\left(r_{i}\right)-y_{\min }\left(r_{i+1}\right)-n(1+\epsilon) \leq-n \epsilon, \quad 1 \leq i \leq T_{d}-1 .
$$

Summing up the above inequality over $2 \leq i \leq T_{d}-1$, together with the inequality $y_{\min }\left(r_{2}\right)-y_{\max }\left(r_{2}\right)<-n(1+\epsilon)$, we obtain

$$
y_{\min }\left(r_{2}\right)-y_{\max }\left(y_{T_{d}}\right)<-n-\epsilon n\left(T_{d}-1\right) .
$$

Further, we have

$$
r_{2}-r_{d}=\left(r_{2}-r_{1}\right)+\left(r_{1}-r_{d}\right) \geq\left\lceil\frac{n}{a}\right\rceil+2+\left(-n-k-\left\lceil\frac{n}{a}\right\rceil-1\right) \geq-n-k+1 .
$$

Combining this with (6.16) and (6.17), we get an estimate

$$
T_{d}<\frac{k}{\epsilon n}+1
$$

This inequality, together with (6.10), gives us the desired bound:

$$
T_{\epsilon}<\frac{\left(\left\lceil\frac{n}{a}\right\rceil+2\right)(k+\epsilon n)}{\epsilon n\left(n+k+\left\lceil\frac{n}{a}\right\rceil+2\right)} .
$$


Step 3. Now fix some $z^{\prime}$. We are going to prove the following upper bound on the number of $i$ with $z_{i}=z^{\prime}$ :

$$
\#\left\{i \mid z_{i}=z^{\prime}\right\} \leq(n+2 a+1)(n+3) .
$$

Denote by $\left(L_{i_{1}}, \ldots, L_{i_{t}}\right)$ the subcollection which consists of bundles with $z$-coordinate $z^{\prime}$ (we assume that $t>0$ ). Also put

$$
\left(p_{j}, q_{j}\right):=\left(x_{i_{j}}, y_{i_{j}}\right), \quad 1 \leq j \leq t .
$$

We need explicit descriptions of one more forbidden sets:

$$
K_{4}=\{(x, y, z) \mid z \geq 0, x \leq-n-2 a-1+\min (0, z-y)\} .
$$

Write down explicitly intersections of (6.3), (6.12), (6.4), (6.5) and (6.20) with the plane $\{z=0\}$ :

$$
\begin{gathered}
M_{\text {eff }}=K_{\text {eff }} \cap\{z=0\}=\{(x, y) \mid x \geq \max (-y, 0)\} ; \\
\widehat{M}_{0}=\widehat{K_{0}} \cap\{z=0\}=\{(x, y) \mid y \geq n+1, x \geq-a y+2 a-1\} ; \\
\widehat{M_{1}}=\widehat{K_{1}} \cap\{z=0\}=\{(x, y) \mid y \geq 1, x \leq-n-2 a-1\} ; \\
\widehat{M}_{2}=\widehat{K_{2}} \cap\{z=0\}=\{(x, y) \mid y \leq-1, x \leq-n-2 a-1-y\} ; \\
M_{4}=K_{4} \cap\{z=0\}=\{(x, y) \mid x \leq-n-2 a-1+\min (0,-y)\} .
\end{gathered}
$$

Also put $M_{\text {all }}:=K_{\text {all }} \cap\{z=0\}$.

From (6.1) we see that

$$
\left(p_{i}-p_{j}, q_{i}-q_{j}\right) \notin M_{\text {all }} \text { for } 1 \leq i<j \leq t .
$$

It is easy to see that

$$
\begin{aligned}
& \{x+y \leq-n-2 a-1\} \subset \widehat{M}_{2} \cup M_{4} \subset M_{\text {all }} ; \\
& \{x+y \geq n+2 a+1\} \subset M_{\text {eff }} \cup \widehat{M}_{0} \subset M_{\text {all }} .
\end{aligned}
$$

Therefore,

$$
\{|x+y| \geq n+2 a+1\} \subset M_{\text {all }}
$$

Further, note that

$$
\{y=-x \leq 0\} \subset M_{\text {eff }} \subset M_{\text {all }}, \quad\{y=-x \geq n+3\} \subset \widehat{M}_{0} \cup \widehat{M}_{1} \subset M_{\text {all }}
$$

(in the second inequality, for $a \geq 2$ the set $\widehat{M}_{1}$ is unnecessary, and $n+3$ can be replaced by $n+1)$. Combining (6.21) with (6.22) and (6.23), we obtain that

$$
\max \left(p_{j}+q_{j}\right)-\min \left(p_{j}+q_{j}\right) \leq n+2 a,
$$


and each line $x+y=d$ contains at most $n+3$ points $\left(p_{i}, q_{i}\right)$. Therefore,

$$
t \leq(n+2 a+1)(n+3),
$$

the desired inequality (6.18) is proved.

Upper bounds (6.2) and (6.18) are yet not sufficient for our purposes.

Step 4. With notation of Step 3, choose some $\epsilon \geq \frac{2 a}{n}$ and make an additional assumption:

$$
\max \left(q_{j}\right)-\min \left(q_{j}\right) \leq n(1+\epsilon)
$$

Under these assumptions, we will obtain another upper bound on $t$ :

$$
\#\left\{i \mid z_{i}=z^{\prime}\right\} \leq\left(\frac{3}{4}+\epsilon\right) n^{2}+\left(\frac{3}{2}+\epsilon+a+2 \epsilon a\right) n-a^{2}+a+1 .
$$

We may and will assume that $\max \left(q_{i}\right)=\max \left(p_{i}+q_{i}\right)=0$. Then for all $1 \leq i \leq t$ we have

$$
\left(p_{i}, q_{i}\right) \in\{-\lfloor n(1+\epsilon)\rfloor \leq q \leq 0,-n-2 a \leq p+q \leq 0\} .
$$

Further, choose indices $b$ and $u$ such that $p_{b}=\min \left(p_{i}\right), p_{u}=\max \left(p_{i}\right)$.

Suppose that $p_{u}-p_{b}>n(1+\epsilon)$. Then we have

$$
\left(p_{b}-p_{u}, q_{b}-q_{u}\right) \in \widehat{M}_{1} \cup \widehat{M}_{2} \cup M_{4} \subset M_{a l l},
$$

since $p_{b}-p_{u}<-n(1+\epsilon)<-n-2 a$ by our assumption. Hence, $b>u$. On the other hand, it follows from (6.26) that

$$
\left(p_{u}-p_{b}, q_{u}-q_{b}\right) \in M_{e f f} \subset M_{a l l},
$$

hence $u>b$, a contradiction.

Therefore, $p_{u}-p_{b} \leq n(1+\epsilon)$, and we have

$$
\begin{aligned}
& \left(p_{i}, q_{i}\right) \in\{-\lfloor n(1+\epsilon)\rfloor \leq q \leq 0,-n-2 a \leq p+q \leq 0, \\
& \left.\qquad p_{b} \leq p \leq p_{b}+\lfloor n(1+\epsilon)\rfloor \cdot\right\}:=Q \subset \mathbb{R}^{2}
\end{aligned}
$$

We are interested in the upper bound on the number of integral points in the polygon $Q$. Denote by $N_{1}$ (resp. $N_{2}$ ) the number of integral points in the interior of $Q$ (resp. on the boundary of $Q$ ). By Pick's Theorem, we have

$$
\operatorname{Area}(Q)=N_{1}+\frac{N_{2}}{2}-1
$$

Hence,

$$
t \leq N_{1}+N_{2}=\operatorname{Area}(Q)+\frac{N_{2}}{2}+1 .
$$


First we make an estimate on $\operatorname{Area}(Q)$. Here we may assume that $p_{b} \leq 0$ (because for $p_{b}>0$ the polygon $Q$ is smaller than for $\left.p_{b}=0\right)$. Then, we have

$$
\begin{aligned}
\operatorname{Area}(Q)=\lfloor n(1+\epsilon)\rfloor(n+2 a)-\frac{1}{2}\left(p_{b}^{2}+\left(n+2 a+p_{b}\right)^{2}\right) \\
\leq(1+\epsilon) n(n+2 a)-\left(\frac{n}{2}+a\right)^{2}=\left(\frac{3}{4}+\epsilon\right) n^{2}+(1+2 \epsilon) a n-a^{2} .
\end{aligned}
$$

Further, it is easy to make an estimate on $N_{2}$. Here we also may assume that $p_{b} \leq 0$, and then

$$
\begin{aligned}
& \text {.30) } N_{2}=\left(-p_{b}\right)+\left(\lfloor n(1+\epsilon)\rfloor+p_{b}\right)+\left(-p_{b}\right)+\left(n+2 a+p_{b}\right) \\
& +\left(\lfloor n(1+\epsilon)\rfloor-n-2 a-p_{b}\right)+\left(n+2 a+p_{b}\right)=2\lfloor n(1+\epsilon)\rfloor+n+2 a \leq(3+2 \epsilon) n+2 a .
\end{aligned}
$$

Combining inequality (6.28) with estimates (6.29) and (6.30), we conclude that

$$
\begin{aligned}
t \leq\left(\frac{3}{4}+\epsilon\right) n^{2}+(1+2 \epsilon) a n-a^{2}+\frac{1}{2}((3 & +2 \epsilon) n+2 a)+1 \\
& =\left(\frac{3}{4}+\epsilon\right) n^{2}+\left(\frac{3}{2}+\epsilon+a+2 \epsilon a\right) n-a^{2}+a+1,
\end{aligned}
$$

the desired inequality (6.25) is proved.

Step 5.

Now we apply estimates (6.2), (6.9), (6.18) and (6.25) to finish the proof. From this moment we assume that $\frac{2 a}{n} \leq \epsilon<\frac{1}{4}$

First, from (6.2), (6.18), (6.25) we obtain the upper bound on the length of our exceptional collection

$$
\begin{aligned}
m \leq T_{\epsilon} \cdot(n+2 a+1)(n+3) & \\
+\left(n+k+\left\lceil\frac{n}{a}\right\rceil\right. & \left.+2-T_{\epsilon}\right)\left(\left(\frac{3}{4}+\epsilon\right) n^{2}+\left(\frac{3}{2}+\epsilon+a+2 \epsilon a\right) n-a^{2}+a+1\right) \\
\leq & T_{\epsilon} \cdot\left(\left(\frac{1}{4}-\epsilon\right) n^{2}+\left(a+\frac{5}{2}\right) n+a^{2}+3 a+2\right) \\
& +\left(n+\left\lceil\frac{n}{a}\right\rceil+k+2\right)\left(\left(\frac{3}{4}+\epsilon\right) n^{2}+\left(\frac{3}{2} a+2\right) n-a^{2}+a+1\right) .
\end{aligned}
$$

Combining (6.31) with (6.9), we get the following inequality:

$$
\begin{aligned}
& m \leq \frac{\left(\left\lceil\frac{n}{a}\right\rceil+2\right)(k+\epsilon n)}{\epsilon n\left(n+\left\lceil\frac{n}{a}\right\rceil+k+2\right)}\left(\left(\frac{1}{4}-\epsilon\right) n^{2}+\left(a+\frac{5}{2}\right) n+a^{2}+3 a+2\right) \\
& +\left(n+\left\lceil\frac{n}{a}\right\rceil+k+2\right)\left(\left(\frac{3}{4}+\epsilon\right) n^{2}+\left(\frac{3}{2} a+2\right) n-a^{2}+a+1\right)=: E(n, k, a, \epsilon) .
\end{aligned}
$$


By the formula (5.1), we have that

$$
\begin{aligned}
\operatorname{rk} K_{0}\left(Y_{n, k, a}\right)=(n+2 a) n+k+ & k n(n+2 a)+n+(n+2 a) k \\
= & n^{2} k+2 a k n+n^{2}+n k+2 a n+2 a k+k+n .
\end{aligned}
$$

Now, combining (6.33) and (6.32), we can write

$$
c \operatorname{rk} K_{0}\left(Y_{n, k, a}\right)-E(n, k, a, \epsilon)=\frac{P_{2}(n, a, \epsilon) k^{2}+P_{1}(n, a, \epsilon) k+P_{0}(n, a, \epsilon)}{\epsilon n\left(n+\left\lceil\frac{n}{a}\right\rceil+k+2\right)},
$$

where

$$
\begin{aligned}
& \text { (6.35) } P_{2}(n, a, \epsilon)=\epsilon n\left(c n(n+2 a)+c n+2 c a+c-\left(\frac{3}{4}+\epsilon\right) n^{2}\right. \\
& \left.-\left(\frac{3}{2} a+2\right) n+a^{2}-a-1\right)=\epsilon n\left(\left(c-\frac{3}{4}-\epsilon\right) n^{2}+\left(2 a c+c-\frac{3}{2} a-2\right) n+2 a c+c+a^{2}-a-1\right) .
\end{aligned}
$$

Now choose some $0<\epsilon<c-\frac{3}{4}$. By (6.35), there exists $n_{0}(a, \epsilon) \geq \frac{2 a}{\epsilon}$ such that for $n \geq n_{0}(a, \epsilon)$ we have $P_{2}(n, a, \epsilon)>0$. Further, for such $n$, according to (6.34), there exists $k_{0}(n, a, \epsilon)>0$ such that for $k \geq k_{0}(n, a, \epsilon)$ we have $c \operatorname{rk} K_{0}\left(Y_{n, k, a}\right)>E(n, k, a, \epsilon)$. Finally, combining with (6.32), we conclude that for such $n, k, a$

$$
l\left(Y_{n, k, a}\right)<c \operatorname{rk} K_{0}\left(Y_{n, k, a}\right) .
$$

Theorem is proved.

Proof of Theorem 6.3. We will apply the proof of the previous Theorem. Namely, by (6.32), we have that

$$
l\left(Y_{16, k, 1}\right) \leq E\left(16, k, 1, \frac{1}{8}\right)
$$

Further, a straightforward computation (solving quadratic inequality in one variable) shows that

$$
\operatorname{rk} K_{0}\left(Y_{16, k, 1}\right)>E\left(16, k, 1, \frac{1}{8}\right), \quad \text { for } k \geq 386 .
$$

This proves Theorem.

7. Strong exceptional collections of length at least $\frac{3}{4} \mathrm{rk} K_{0}(Y)$.

In this section we prove the following theorem.

Theorem 7.1. For any toric nef-Fano DM stack $Y$ with Picard number three, there exists a strong exceptional collection of line bundles on $Y$ of length at least $\frac{3}{4} \mathrm{rk} K_{0}(Y)$. 
Proof. Let $\Sigma$ be a fan describing $Y$. Then by Appendix, Theorem A.1, there exists a number $t \in \mathbb{Z}_{>0}$ and a decomposition

$$
\Sigma(1)=\bigsqcup_{i \in \mathbb{Z} /(2 t+1)} X_{i}
$$

with $X_{i} \neq \emptyset$, such that the primitive collections are precisely

$$
X_{i} \cup X_{i+1} \cup \cdots \cup X_{i+t-1}, \quad i \in \mathbb{Z} /(2 t+1) \mathbb{Z} .
$$

Denote by $K_{i}$ (resp. $\widehat{K}_{i}$ ), $i \in \mathbb{Z} /(2 t+1)$, the forbidden set corresponding to $X_{i} \cup X_{i+1} \cup$ $\cdots \cup X_{i+t}$ (resp. $X_{i-1} \cup X_{i-2} \cup \cdots \cup X_{i-t}$ ), and by $K_{n e g}$ the forbidden set corresponding to $\Sigma(1)$. Also put

$$
K_{\text {bad }}:=\bigcup_{i \in \mathbb{Z} /(2 t+1)}\left(K_{i} \cup \widehat{K_{i}}\right) \cup K_{\text {neg }}
$$

Then $K_{b a d}$ is the set of all line bundles with non-zero higher cohomology (by Corollary 4.2 and Appendix, Theorem A.1).

Denote by $E_{i} \in \operatorname{Pic} Y, i \in \Sigma(1)$, the invariant divisors. Put

$$
\widehat{\operatorname{Pic}}_{\mathbb{R}}(Y):=\operatorname{Pic}_{\mathbb{R}}(Y) /\left(\mathbb{R} \cdot K_{Y}\right) .
$$

Denote by $\pi: \operatorname{Pic}_{\mathbb{R}}(Y) \rightarrow \widehat{\operatorname{Pic}}_{\mathbb{R}}(Y)$ the projection, and by $\iota: \operatorname{Pic}(Y) \rightarrow \operatorname{Pic}_{\mathbb{R}}(Y)$ the inclusion. We will often write $E_{i}$ instead of $\iota\left(E_{i}\right)$. Put $\widehat{E_{i}}:=\pi\left(E_{i}\right)$.

Take the polytope

$$
\widehat{P}:=\sum_{i \in \Sigma(1)}\left[0, \widehat{E}_{j}\right] \subset \widehat{\mathrm{Pic}}_{\mathbb{R}}(Y)
$$

the Minkowski sum of the intervals $\left[0, \widehat{E_{j}}\right]$. It is easy to see that $\widehat{P}$ is centrally symmetric with respect to zero (since $\left.\sum_{i \in \Sigma(1)} \widehat{E}_{i}=0\right)$. Further, fix some functional $l: \operatorname{Pic}_{\mathbb{R}}(Y) \rightarrow \mathbb{R}$ such that $l\left(E_{i}\right)>0$ for $i \in \Sigma(1)$. Consider the polytope

$$
P:=\left\{v \in \operatorname{Pic}_{\mathbb{R}}(Y)|\pi(v) \in \widehat{P},| l(v) \mid \leq l\left(-K_{Y}\right)\right\} \subset \operatorname{Pic}_{\mathbb{R}}(Y) .
$$

It is also centrally symmetric (since $\widehat{P}$ is). Denote by $\operatorname{Int}(P)$ the interior of $P$.

Lemma 7.2. For each $p \in \operatorname{Pic}_{\mathbb{R}}(Y)$, the set

$$
\iota^{-1}\left(p+\frac{1}{2} \operatorname{Int}(P)\right) \subset \operatorname{Pic}(Y)
$$

can be ordered in such a way that it becomes a strong exceptional collection.

Proof. It suffices to prove that for any $L_{1}, L_{2} \in \iota^{-1}\left(p+\frac{1}{2} \operatorname{Int}(P)\right)$ we have $H^{>0}\left(L_{2} L_{1}^{-1}\right)=0$. Further, this would follow from the absence of intersection:

$$
\operatorname{Int}(P) \cap \iota\left(K_{\text {bad }}\right)=\emptyset .
$$


Now, choose some functionals $l_{i}: \widehat{\operatorname{Pic}}_{\mathbb{R}}(Y) \rightarrow \mathbb{R}, \quad i \in \mathbb{Z} /(2 t+1)$, such that

$$
l_{i}\left(\widehat{E_{j}}\right) \begin{cases}\geq 0 & \text { for } j \in X_{i} \cup X_{i+1} \cup \cdots \cup X_{i+t} ; \\ \leq 0 & \text { for } j \in X_{i-1} \cup X_{i-2} \cup \cdots \cup X_{i-t}\end{cases}
$$

(they exist by Appendix, Theorem A.1). Then

$$
\pi^{*} l_{i}(\iota(D)) \leq l_{i}\left(-\sum_{j \in X_{i} \cup X_{i+1} \cup \cdots \cup X_{i+t}} \widehat{E_{j}}\right) \quad \text { for } D \in K_{i},
$$

and

$$
\pi^{*} l_{i}(v)>l_{i}\left(-\sum_{j \in X_{i} \cup X_{i+1} \cup \cdots \cup X_{i+t}} \widehat{E_{j}}\right) \quad \text { for } v \in \operatorname{Int}(P) .
$$

Therefore, $\operatorname{Int}(P) \cap \iota\left(K_{i}\right)=\emptyset$. Analogously, $\operatorname{Int}(P) \cap \iota\left(\widehat{K_{i}}\right)=\emptyset$. Finally, we have

$$
l(\iota(D)) \leq l\left(K_{Y}\right) \quad \text { for } D \in K_{n e g},
$$

and

$$
l(v)>l\left(K_{Y}\right) \quad \text { for } v \in \operatorname{Int}(P) .
$$

Therefore, $\operatorname{Int}(P) \cap \iota\left(K_{\text {neg }}\right)=\emptyset$. This proves (7.3). Lemma is proved.

Fix the volume form $\omega$ on $\operatorname{Pic}_{\mathbb{R}}(Y)$ such that $\left|\omega\left(D_{1}, D_{2}, D_{3}\right)\right|=|\operatorname{Ker}(\iota)|$ for any $\mathbb{Z}$ basis $\left(D_{1}, D_{2}, D_{3}\right)$ of $\iota(\operatorname{Pic}(Y))$. Below we take volumes with respect to this form.

Lemma 7.3. There exists a point $p \in \mathrm{Pic}_{\mathbb{R}}$ such that

$$
\left|\iota^{-1}\left(p+\frac{1}{2} \operatorname{Int}(P)\right)\right| \geq \frac{1}{8} \operatorname{Vol}(P) .
$$

Proof. This follows easily from Fubini's theorem. Namely, fix some $\mathbb{Z}$-basis $\left(D_{1}, D_{2}, D_{3}\right)$ of $\iota(\operatorname{Pic}(Y))$. Put $C:=[0,1)^{3}$, and take the measure $d x \wedge d y \wedge d z$ on $C$. Further, take a measure on $\mathbb{Z}^{3}$ such that the measure of each point equals to $|\operatorname{Ker}(\iota)|$. Then we have an isomorphism of spaces with measure:

$C \times \mathbb{Z}^{3} \stackrel{\sim}{\rightarrow} \operatorname{Pic}_{\mathbb{R}}(Y),\left(\left(t_{1}, t_{2}, t_{3}\right),\left(m_{1}, m_{2}, m_{3}\right)\right) \mapsto\left(t_{1}+m_{1}\right) D_{1}+\left(t_{2}+m_{2}\right) D_{2}+\left(t_{3}+m_{3}\right) D_{3}$.

Denote by $q: \operatorname{Pic}_{\mathbb{R}}(Y) \rightarrow C$ the resulting projection. Then, by Fubini's theorem,

$$
\frac{1}{8} \operatorname{Vol}(P)=\int_{C} \mid\left(|\operatorname{Ker}(\iota)| \cdot\left|q^{-1}\left(t_{1}, t_{2}, t_{3}\right) \cap \frac{1}{2} \operatorname{Int}(P)\right|\right) .
$$

Since $\operatorname{Vol}(C)=1$, there exists $\left(t_{1}, t_{2}, t_{3}\right) \in C$ such that

$$
\frac{1}{8} \operatorname{Vol}(P) \leq|\operatorname{Ker}(\iota)| \cdot\left|q^{-1}\left(t_{1}, t_{2}, t_{3}\right) \cap \frac{1}{2} \operatorname{Int}(P)\right|=\left|\iota^{-1}\left(t_{1} D_{1}+t_{2} D_{2}+t_{3} D_{3}+\frac{1}{2} \operatorname{Int}(P)\right)\right| \text {. }
$$

Hence, (7.4) holds for $p=t_{1} D_{1}+t_{2} D_{2}+t_{3} D_{3}$. Lemma is proved.

Now we will obtain the lower bound on $\operatorname{Vol}(P)$. 
Lemma 7.4. The following inequality holds:

$$
\operatorname{Vol}(P) \geq 6 \operatorname{rk} K_{0}(Y) .
$$

Proof. Take the volume form $\widehat{\omega}$ on $\widehat{\operatorname{Pic}}_{\mathbb{R}}(Y)$ such that

$$
\omega=\pi^{*}(\widehat{\omega}) \wedge d l .
$$

Using the form $\widehat{\omega}$ we identify $\Lambda^{2}\left(\widehat{\operatorname{Pic}}_{\mathbb{R}}(Y)\right) \cong \mathbb{R}$. Then for any $G_{1}, G_{2}, G_{3} \in \operatorname{Pic}_{\mathbb{R}}(Y)$ we have

$$
\omega\left(G_{1}, G_{2}, G_{3}\right)=l\left(G_{1}\right) \pi\left(G_{2}\right) \wedge \pi\left(G_{3}\right)+l\left(G_{2}\right) \pi\left(G_{3}\right) \wedge \pi\left(G_{1}\right)+l\left(G_{3}\right) \pi\left(G_{1}\right) \wedge \pi\left(G_{2}\right) .
$$

Now put

$$
W_{i}:=\sum_{j \in X_{i}} E_{j}, \quad i \in \mathbb{Z} /(2 t+1) .
$$

Put $\widehat{W}_{i}:=\pi\left(W_{i}\right)$. We may and will assume that

$$
\widehat{W}_{i} \wedge \widehat{W}_{i+j} \geq 0 \text { for } i \in \mathbb{Z} /(2 t+1), 1 \leq j \leq t
$$

(otherwise we multiply $\omega$ and $\widehat{\omega}$ by $(-1)$ ). Then we have

$$
\left(\widehat{W}_{i}+\cdots+\widehat{W}_{i+t}\right) \wedge\left(\widehat{W}_{i+1}+\cdots+\widehat{W}_{i+t}\right)=\sum_{j=1}^{t} \widehat{W}_{i} \wedge \widehat{W}_{i+j} \geq 0 .
$$

Analogously,

$$
\left(\widehat{W}_{i}+\cdots+\widehat{W}_{i+t-1}\right) \wedge\left(\widehat{W}_{i}+\cdots+\widehat{W}_{i+t}\right) \geq 0 .
$$

It follows from (7.7) and (7.8) that

$$
\begin{aligned}
\operatorname{Vol}(\widehat{P}) \geq \sum_{i \in \mathbb{Z} /(2 t+1)} \frac{1}{2}\left(\left(\widehat{W}_{i}+\cdots+\widehat{W}_{i+t}\right) \wedge\left(\widehat{W}_{i+1}+\cdots+\widehat{W}_{i+t}\right)\right. \\
\left.\quad+\left(\widehat{W}_{i}+\cdots+\widehat{W}_{i+t-1}\right) \wedge\left(\widehat{W}_{i}+\cdots+\widehat{W}_{i+t}\right)\right)=\sum_{\substack{i \in \mathbb{Z} /(2 t+1), 1 \leq j \leq t}} \widehat{W}_{i} \wedge \widehat{W}_{i+j} .
\end{aligned}
$$

Hence,

$$
\operatorname{Vol}(P)=2 l\left(-K_{Y}\right) \operatorname{Vol}(\widehat{P}) \geq 2\left(\sum_{i \in \mathbb{Z} /(2 t+1)} l\left(W_{i}\right)\right)\left(\sum_{\substack{i \in \mathbb{Z} /(2 t+1), 1 \leq j \leq t}} \widehat{W}_{i} \wedge \widehat{W}_{i+j}\right) .
$$

We are going to obtain a similar upper bound on $\operatorname{rk} K_{0}(Y)$. From (3.1), Lemma 2.6 and Lemma 2.7 it follows that $\mathrm{rk} K_{0}(Y)$ equals to the sum of $\left|\omega\left(E_{u_{1}}, E_{u_{2}}, E_{u_{3}}\right)\right|$ over all subsets $\left\{u_{1}, u_{2}, u_{3}\right\} \subset \Sigma(1)$ which are complements to the maximal cones. These are precisely sets $\left\{u_{1}, u_{2}, u_{3}\right\} \subset \Sigma(1)$. such that for some $i \in \mathbb{Z} /(2 t+1), \quad 1 \leq j_{1}, j_{2} \leq t, j_{1}+j_{2}>t$ we have

$$
u_{1} \in X_{i}, \quad u_{2} \in X_{i+j_{1}}, \quad u_{3} \in X_{i-j_{2}} .
$$


Therefore, we have

$$
\operatorname{rk} K_{0}(Y)=\sum_{i \in \mathbb{Z} /(2 t+1)} l\left(W_{i}\right)\left(\sum_{\substack{1 \leq j_{1}, j_{2} \leq t, j_{1}+j_{2}>t}} \widehat{W}_{i+j_{1}} \wedge \widehat{W}_{i-j_{2}}\right) .
$$

Sublemma. Suppose that we are given with collection of vectors $g_{i} \in \widehat{\operatorname{Pic}}_{\mathbb{R}}(Y), i \in$ $\mathbb{Z} /(2 t+1)$, with $\sum_{i \in \mathbb{Z} /(2 t+1)} g_{i}=0$. Suppose that that there exist non-zero functionals $f_{i}$ : $\widehat{\operatorname{Pic}}_{\mathbb{R}}(Y) \rightarrow \mathbb{R}$ such that

$$
f_{i}\left(g_{j}\right) \begin{cases}\geq 0 & \text { for } j=i, i+1, \ldots, i+t \\ \leq 0 & \text { for } j=i-1, i-2, \ldots, i-t\end{cases}
$$

Assume that

$$
g_{i} \wedge g_{i+j} \geq 0 \quad \text { for } i \in \mathbb{Z} /(2 t+1), 1 \leq j \leq t
$$

Then

$$
\sum_{\substack{r \in \mathbb{Z} /(2 t+1), 1 \leq j \leq t}} g_{r} \wedge g_{r+j} \geq 3 \sum_{\substack{1 \leq j_{1}, j_{2} \leq t, j_{1}+j_{2}>t}} g_{j_{1}} \wedge g_{-j_{2}}
$$

Proof. First note that

$$
\sum_{\substack{1 \leq j_{1}, j_{2} \leq t, j_{1}+j_{2}>t}} g_{j_{1}} \wedge g_{-j_{2}}=\sum_{j=1}^{t}\left(g_{-j} \wedge\left(\sum_{k=1}^{t} g_{k-j}+\sum_{k=j+1}^{t} g_{-k}\right)\right)=\sum_{\substack{1 \leq j_{1} \leq t, 0 \leq j_{2} \leq t-j_{1}}} g_{-j_{1}} \wedge g_{j_{2}} .
$$

Similarly,

Therefore,

$$
\sum_{\substack{1 \leq j_{1}, j_{2} \leq t, j_{1}+j_{2}>t}} g_{j_{1}} \wedge g_{-j_{2}}=\sum_{\substack{0 \leq j_{1} \leq t, 1 \leq j_{2} \leq t-j_{1}}} g_{-j_{1}} \wedge g_{j_{2}}
$$

$$
\begin{aligned}
\sum_{\substack{r \in \mathbb{Z} /(2 t+1), 1 \leq j \leq t}} g_{r} \wedge g_{r+j}-3 \sum_{\substack{1 \leq j_{1}, j_{2} \leq t, j_{1}+j_{2}>t}} g_{j_{1}} \wedge g_{-j_{2}}=\sum_{\substack{r \in \mathbb{Z} /(2 t+1), 1 \leq j \leq t}} g_{r} \wedge g_{r+j}-\sum_{\substack{1 \leq j_{1}, j_{2} \leq t, j_{1}+j_{2}>t}} g_{j_{1}} \wedge g_{-j_{2}} \\
-\sum_{\substack{1 \leq j_{1} \leq t, 0 \leq j_{2} \leq t-j_{1}}} g_{-j_{1}} \wedge g_{j_{2}}-\sum_{\substack{0 \leq j_{1} \leq t, 1 \leq j_{2} \leq t-j_{1}}} g_{-j_{1}} \wedge g_{j_{2}} \\
=\sum_{1 \leq j_{1}<j_{2} \leq t} g_{-j_{2}} \wedge g_{-j_{1}}+\sum_{\substack{1 \leq j_{1}<j_{2} \leq t \\
j_{1}}} g_{j_{1}} \wedge g_{j_{2}}-\sum_{\substack{1 \leq j_{1} \leq t, 1 \leq j_{2} \leq t-j_{1}}} g_{-j_{1}} \wedge g_{j_{2}} .
\end{aligned}
$$

Thus, we are left to prove the following inequality:

$$
\sum_{1 \leq j_{1}<j_{2} \leq t} g_{-j_{2}} \wedge g_{-j_{1}}+\sum_{1 \leq j_{1}<j_{2} \leq t} g_{j_{1}} \wedge g_{j_{2}}-\sum_{\substack{1 \leq j_{1} \leq t, 1 \leq j_{2} \leq t-j_{1}}} g_{-j_{1}} \wedge g_{j_{2}} \geq 0 .
$$


We proceed by induction on $t$. For $t=1$, the LHS of (7.13) equals to zero, and there is nothing to prove.

Suppose that (17.13) is proved in the case $t \leq m$. Let us prove it for $t=m+1$. Consider the cases

Case 1: for some $j \neq 0$ we have $g_{j}=0$. We may and will assume that $j \in\{1, \ldots, t\}$ (by the symmetry). Form another collection $g_{i}^{\prime} \in \widehat{\operatorname{Pic}}_{\mathbb{R}}(Y), i \in \mathbb{Z} /(2 t-1)$, given by the formula

$$
\left(g_{0}^{\prime}, g_{1}^{\prime}, \ldots, g_{2 t-2}^{\prime}\right)=\left(g_{0}, g_{1}, \ldots, g_{j-1}, g_{j+1}, \ldots, g_{j+t}+g_{j+t+1}, \ldots, g_{2 t}\right)
$$

(in the case $j=t$ we have $\left.g_{0}^{\prime}=g_{2 t}+g_{2 t+1}\right)$. Clearly, this new collection satisfies the assumptions of Sublemma, and one computes that

$$
\begin{gathered}
\left(\sum_{1 \leq j_{1}<j_{2} \leq t} g_{-j_{2}} \wedge g_{-j_{1}}+\sum_{1 \leq j_{1}<j_{2} \leq t} g_{j_{1}} \wedge g_{j_{2}}-\sum_{\substack{1 \leq j_{1} \leq t, j_{1} \\
1 \leq j_{2} \leq t-j_{1}}} g_{-j_{1}} \wedge g_{j_{2}}\right)- \\
-\left(\sum_{1 \leq j_{1}<j_{2} \leq t-1} g_{-j_{2}}^{\prime} \wedge g_{-j_{1}}^{\prime}+\sum_{1 \leq j_{1}<j_{2} \leq t-1} g_{j_{1}}^{\prime} \wedge g_{j_{2}}^{\prime}-\sum_{\substack{1 \leq j_{1} \leq t-1, 1 \leq j_{2} \leq t-1-j_{1}}} g_{-j_{1}}^{\prime} \wedge g_{j_{2}}^{\prime}\right)= \\
=g_{j+t} \wedge g_{j+t+1} \geq 0 .
\end{gathered}
$$

Then, inequality (7.13) follows from (7.14) and inductive hypothesis.

Case 2: we have $g_{j} \neq 0$ for $j \neq 0$, but for some $j \neq 0, t+1$ we have $g_{j}=-\kappa g_{t+j}$, $\kappa>0$. We may and will assume that $\kappa \geq 1$. We form another collection $g_{i}^{\prime} \in \widehat{\operatorname{Pic}}_{\mathbb{R}}(Y)$, $i \in \mathbb{Z} /(2 t+1)$, given by the formula

$$
g_{i}^{\prime}:= \begin{cases}g_{j}+g_{t+j} & \text { for } i=j \\ 0 & \text { for } i=j+t \\ g_{i} & \text { for } i \in(\mathbb{Z} /(2 t+1)) \backslash\{j, j+t\} .\end{cases}
$$

This new collection obviously satisfies the assumptions of Sublemma and we have

$$
\begin{aligned}
& \left(\sum_{1 \leq j_{1}<j_{2} \leq t} g_{-j_{2}} \wedge g_{-j_{1}}+\sum_{1 \leq j_{1}<j_{2} \leq t} g_{j_{1}} \wedge g_{j_{2}}-\sum_{\substack{1 \leq j_{1} \leq t, 1 \leq j_{2} \leq t-j_{1}}} g_{-j_{1}} \wedge g_{j_{2}}\right)- \\
& -\left(\sum_{1 \leq j_{1}<j_{2} \leq t} g_{-j_{2}}^{\prime} \wedge g_{-j_{1}}^{\prime}+\sum_{1 \leq j_{1}<j_{2} \leq t} g_{j_{1}}^{\prime} \wedge g_{j_{2}}^{\prime}-\sum_{\substack{1 \leq j_{1} \leq t, 1 \leq j_{2} \leq t-j_{1}}} g_{-j_{1}}^{\prime} \wedge g_{j_{2}}^{\prime}\right)= \\
& \quad=\left\{\begin{array}{l}
\left(g_{j+1}+\cdots+g_{j+t-1}\right) \wedge g_{j+t} \geq 0 \quad \text { if } j \in\{1, \ldots, t\} ; \\
g_{j+t} \wedge\left(g_{j-1}+\cdots+g_{j-t}\right) \geq 0 \quad \text { if } j \in\{t+2, \ldots, 2 t\} .
\end{array}\right.
\end{aligned}
$$

Hence, we are reduced to the Case 1. 
We are left with

Case 3: we have that $g_{j}, j \neq 0$, are pairwise linearly independent. Take (unique) $\kappa>0$ such that $g_{2 t}+\kappa g_{t}$ is linearly dependent with $g_{t-1}$. Put

$$
\kappa^{\prime}:=\min (\kappa, 1)
$$

Form another collection $g_{i}^{\prime} \in \widehat{\operatorname{Pic}}_{\mathbb{R}}(Y), \quad i \in \mathbb{Z} /(2 t+1)$, given by the formula

$$
g_{i}^{\prime}:= \begin{cases}g_{2 t}+\kappa^{\prime} g_{t} & \text { for } i=2 t \\ \left(1-\kappa^{\prime}\right) g_{t} & \text { for } i=t \\ g_{i} & \text { for } i \in(\mathbb{Z} /(2 t+1)) \backslash\{t, 2 t\} .\end{cases}
$$

This new collection obviously satisfies the assumptions of Sublemma and we have

$$
\begin{aligned}
& \left(\sum_{1 \leq j_{1}<j_{2} \leq t} g_{-j_{2}} \wedge g_{-j_{1}}+\sum_{1 \leq j_{1}<j_{2} \leq t} g_{j_{1}} \wedge g_{j_{2}}-\sum_{\substack{1 \leq j_{1} \leq t, 1 \leq j_{2} \leq t-j_{1}}} g_{-j_{1}} \wedge g_{j_{2}}\right)- \\
& -\left(\sum_{1 \leq j_{1}<j_{2} \leq t} g_{-j_{2}}^{\prime} \wedge g_{-j_{1}}^{\prime}+\sum_{1 \leq j_{1}<j_{2} \leq t} g_{j_{1}}^{\prime} \wedge g_{j_{2}}^{\prime}-\sum_{\substack{1 \leq j_{1} \leq t, 1 \leq j_{2} \leq t-j_{1}}} g_{-j_{1}}^{\prime} \wedge g_{j_{2}}^{\prime}\right)= \\
& =\kappa^{\prime} g_{t} \wedge\left(g_{t+1}+\cdots+g_{2 t-1}\right) \geq 0 .
\end{aligned}
$$

We are reduced either to the Case 1 (if $\kappa^{\prime}=1$ ) or to the Case 2 (if $\kappa^{\prime}<1$ ).

In all cases the inductive statement is proved for $t=m+1$. Sublemma is proved.

Finally, from (7.10), Sublemma and (7.11) we get the following chain of equalities and inequalities:

$$
\begin{aligned}
\operatorname{Vol}(P) \geq 2\left(\sum_{i \in \mathbb{Z} /(2 t+1)} l\left(W_{i}\right)\right) & \left(\sum_{\substack{i \in \mathbb{Z} /(2 t+1), 1 \leq j \leq t}} \widehat{W}_{i} \wedge \widehat{W}_{i+j}\right) \\
& \geq 6 \sum_{i \in \mathbb{Z} /(2 t+1)} l\left(W_{i}\right)\left(\sum_{\substack{1 \leq j_{1}, j_{2} \leq t, j_{1}+j_{2}>t}} \widehat{W}_{i+j_{1}} \wedge \widehat{W}_{i-j_{2}}\right)=6 \operatorname{rk} K_{0}(Y) .
\end{aligned}
$$

Lemma is proved.

From Lemmas 7.2, 7.3 and 7.4 it follows that for some $p \in \operatorname{Pic}(\mathbb{R})$ the set $\iota^{-1}\left(p+\frac{1}{2} \operatorname{Int}(P)\right)$ can be ordered in such a way that it becomes a strong exceptional collection of line bundles of length at least $\frac{1}{8} \cdot 6 \mathrm{rk} K_{0}(Y)=\frac{3}{4} \mathrm{rk} K_{0}(Y)$. Theorem is proved. 


\section{Appendix A. Smooth projective toric DM stacks with Picard number three}

Here we describe combinatorial structure of the fans defining smooth projective toric DM stacks with Picard number three. Let $Y$ be such a stack, $\Sigma$ a fan in a lattice $N$ defining it, and $v_{i} \in N, i \in \Sigma(1)$ are marked vectors on one-dimensional cones. Denote by $E_{i} \in \operatorname{Pic}(Y), \quad i \in \Sigma(1)$, the invariant divisors.

Theorem A.1. 1) There exists $t \geq 1$ and a decomposition

$$
\Sigma(1)=\bigsqcup_{i \in \mathbb{Z} /(2 t+1)} X_{i}
$$

such that the primitive collections are precisely

$$
X_{i} \cup X_{i+1} \cup \cdots \cup X_{i+t-1}, \quad i \in \mathbb{Z} /(2 t+1) .
$$

2) If $Y$ is Fano (resp. nef-Fano) then there exist non-zero functionals $l_{i}: \operatorname{Pic}_{\mathbb{R}}(Y) \rightarrow \mathbb{R}$, $i \in \mathbb{Z} /(2 t+1)$ such that $l_{i}\left(K_{Y}\right)=0$ and $l_{i}\left(E_{j}\right)>0$ (resp. $\left.l_{i}\left(E_{j}\right) \geq 0\right)$ for $j \in X_{i} \cup$ $X_{i+1} \cup \cdots \cup X_{i+t}$, and $l_{i}\left(E_{j}\right)<0$ (resp. $l_{i}\left(E_{j}\right) \leq 0$ ) for $j \in X_{i-1} \cup X_{i-2} \cup \cdots \cup X_{i-t}$.

3) The subsets $I \subset \Sigma(1)$ such that $\left|C_{I}\right|$ has non-trivial reduced homology are precisely the following: $\emptyset, \quad \Sigma(1), \quad X_{i} \cup X_{i+1} \cup \cdots \cup X_{i+t}, \quad X_{i-1} \cup X_{i-2} \cup \cdots \cup X_{i-t}, \quad i \in \mathbb{Z} /(2 t+1)$.

Proof. 1) Take any $\mathbb{Q}$-ample line bundle $L \in \operatorname{Pic}(Y)$. Then by [FLTZ] (Theorem 4.4), it can be written as

$$
L=\sum_{i \in \Sigma(1)} a_{i} E_{i} \in \operatorname{Pic}_{\mathbb{Q}}(Y), \quad a_{i} \in \mathbb{Q}_{>0}
$$

and the polytope

$$
\bigcup_{\left\langle v_{i_{1}}, \ldots, v_{i_{\operatorname{dim}} Y}\right\rangle \in \Sigma(\operatorname{dim} Y)} \operatorname{conv}\left(\frac{v_{i_{1}}}{a_{1}}, \ldots, \frac{v_{i_{\operatorname{dim} Y}}}{a_{\operatorname{dim} Y}}, 0\right)
$$

is convex, with vertices being precisely all $v_{i}$.

Denote by $\pi: \operatorname{Pic}_{\mathbb{R}}(Y) \rightarrow \operatorname{Pic}_{\mathbb{R}}(Y) /(\mathbb{R} \cdot L)$ the projection.

For each set $\left\{u_{1}, u_{2}, u_{3}\right\} \subset \Sigma(1)$ which is a completion to some maximal cone, we have by Proposition 2.4

$$
0 \in \mathbb{R}_{>0} \pi\left(E_{u_{1}}\right)+\mathbb{R}_{>0} \pi\left(E_{u_{2}}\right)+\mathbb{R}_{>0} \pi\left(E_{u_{3}}\right) .
$$

Moreover, for any $u_{1}, u_{2} \in \Sigma(1)$ we have again by Proposition 2.4

$$
0 \notin \mathbb{R}_{>0} \pi\left(E_{u_{1}}\right)+\mathbb{R}_{>0} \pi\left(E_{u_{2}}\right) .
$$

Now the desired subsets $X_{i} \subset \Sigma(1)$ are defined as maximal subsets $X \subset \Sigma(1)$ with the following property:

$$
-E_{k} \notin \sum_{j \in X} \mathbb{R}_{\geq 0} E_{j}=: A_{X} \quad \text { for each } k \in \Sigma(1) .
$$


It follows from (A.3) and (A.2) that:

(i) the number of such $X$ is odd and at least three, say $2 t+1$;

(ii) they are disjunctive and their union is $\Sigma(1)$;

(iii) for different $X_{i}$ and $X_{j}$ we have that $A_{X_{i}} \cap A_{X_{j}}=\{0\}$.

We order the $X_{i}$ cyclically in such a way that the cones $A_{X_{0}}, \ldots A_{X_{2 t}}$ go in the anticlockwise direction (for some orientation on $\operatorname{Pic}_{\mathbb{R}}(Y) /(\mathbb{R} \cdot L)$ ). It is clear from (A.2) that primitive collections are precisely as in (A.1).

2) If $Y$ is Fano, then the statement follows from the proof of 1). Let $Y$ be nef-Fano. Then there exist sequence $a_{\sigma, n}>0$, with $\sigma \in \Sigma(1), n \geq 1$, such that

$$
\lim _{n \rightarrow \infty} a_{\sigma, n}=1, \quad \text { and } \quad L_{n}=\sum_{\sigma \in \Sigma(1)} a_{\sigma, n} E_{\sigma} \text { is } \mathbb{Q}-\text { ample for all } n .
$$

Denote by $l_{i, n}, \quad i \in \mathbb{Z} /(2 t+1)$, the desired functionals for $L_{n}$ instead of $-K_{Y}$. Assume that $\left\|l_{i, n}\right\|=1$ for some norm on $\operatorname{Pic}_{\mathbb{R}}(Y)$. Then each sequence $\left\{l_{i, n}\right\}_{n=1}^{\infty}$ has some partial limit $l_{i}$. The functionals $l_{i}$ satisfy the desired properties.

3) It suffices to remind that the following holds:

(iv) if $\bar{H}$. $\left(C_{I}\right) \neq 0$ and $I \neq \emptyset$, then $I$ is a union of primitive collections (Lemma 4.4);

(v) if $\bar{H} .\left(C_{I}\right) \neq 0$, then also $\bar{H} \cdot\left(C_{\Sigma(1) \backslash I}\right) \neq 0$.

(vi) if $I$ is a primitive collection, then $\bar{H} \cdot\left(C_{I}\right) \neq 0$.

The assertion immediately follows from (iv), (v), (vi) and part 1).

\section{REFERENCES}

[Ba] V. V. Batyrev, On the classification of smooth projective toric varieties, Tohoku Math. J. 43 (1991), 569-585.

[BHu] L. Borisov, Z. Hua, On the conjecture of King for smooth toric Deligne-Mumford stacks, Adv. Math. Volume 221, Issue 1 (2009), pp. 277-301.

[BHo] L. Borisov, R.P. Horja, Mellin-Barnes integrals as Fourier-Mukai transforms, Adv. Math. 207 (2006), no. 2, pp. 876-927.

[CM] L. Costa, R.M. Miró-Roig, Tilting sheaves on toric varieties. Math. Z., 248:849-865, 2004.

[FLTZ] B. Fang, Chiu-Chu Melissa Liu, D. Treumann, E. Zaslow, The Coherent-Constructible Correspondence for Toric Deligne-Mumford Stacks, aXiv:0911.4711 (preprint).

[HP1] L. Hille, M. Perling, A counterexample to King's conjecture. Compos. Math. 142 (2006), no. 6, 1507-1521.

[HP2] L. Hille, M. Perling, Exceptional sequences of invertible sheaves on rational surfaces, to appear in Compos. Math., arXiv:0810.1936 (preprint).

[IU] A. Ishii, K. Ueda, Dimer models and exceptional collections, arXiv:0911.4529 (preprint).

[Ka] Y. Kawamata, Derived categories of toric varieties, Michigan Math. J. 54 (2006), no. 3, 517-535.

[Ki] A. D. King, Tilting bundles on some rational surfaces. preprint, 1997.

[Mi] M. Michalek, A family of counter-examples to King's conjecture, arXiv:1009.0821 (preprint). 
Steklov Mathematical Institute of RAS, Gubkin str. 8, GSP-1, Moscow 119991, Russia

E-mail address: efimov@mccme.ru 